\title{
Muscular strength profile in Tunisian male national judo team
}

\author{
Mourad Ghrairi ${ }^{1,2,4}$ \\ Omar Hammouda ${ }^{1}$ \\ Nikos Malliaropoulos 3,4
}

1 National Center of Sciences and Sports Medicine, Tunis, Tunisia

2 Garhoud Hospital \& Al Shabab Sport Club, Sports Medicine Clinic, Dubai UAE

3 National Track and Field Center, Sports Injury Clinic, SEGAS, Thessaloniki, Greece

4 European College of Sports and Exercise Physicians ECOSEP

Corresponding author:

Mourad Ghrairi

Garhoud Hospital \& Al Shabab Sport Club, Sports

Medicine Clinic, Dubai

PO Box 3458 Dubai UAE

Phone: 00.971.50.6550507

Fax: 00.971.4.2966605

E-mail:drmg57@yahoo.com

\section{Summary}

Background: it is well established that muscle strength is a determinant factor in judo. However, little data are available for African athletes. Therefore, the aim of this study was to provide reference data of the muscular strength profile (MSP) for an African team, Tunisian judo team.

Methods: the study was conducted among ten international judo athletes from Tunisia. To determine their MSP, we used an isokinetic dynamometer to assess Hamstrings, Quadriceps of both knees and external, internal rotators of both shoulders. The angular velocities of the assessments were; 90, 180, $240 \%$ for the knees and $60,120 \%$ for the shoulders. Results: MSP was determined based on two parameters; the maximum peak torque (PT) of each muscle and the ratio agonistic/antagonistic muscles (R). The knee extensors and flexors in the "supporting leg" had higher PT than in the "attacking leg"; respectively, 245N.m versus 237 $(p<0.05)$ and 147 N.m versus $145(p>0.05)$. R was normal for both legs. Furthermore, both rotators of the dominant shoulder had higher PT; 84 N.m versus 71 for the internal rotators $(p<0.05)$ and 34,7 N.m versus 29,0 for the lateral rotators $(p<0.05)$. Inversely, $R$ was higher in the non-dominant side; $45 \%$ versus $35, \mathrm{p}<0.05$ ).

Conclusion: the MSP of the selected elites Tunisian judo athletes was characterized by 3 major features; a strength of the quadriceps in the standing leg significantly higher than in the attacking leg, a normal muscular balance Hamstrings/quadriceps in both legs and a strength of the shoulder' rotators higher in the dominant side.

KEY WORDS: Judo, isokinetic, knee, shoulder, muscular imbalance.

\section{Introduction}

Judo is a dynamic, high intensity intermittent sport that requires complex skills and tactical excellence for success ${ }^{1-3}$. To be successful in international competitions, judo athletes must achieve an excellent level of physical fitness and condition during training, where the muscular strength plays one of the most important roles ${ }^{3}$. This determinant physical factor during combat has different parameters (i.e., maximal strength, power, endurance, fatigue index). These can be measured with many devices, but the most reliable of which is the computerized isokinetic dynamometry ${ }^{4-9}$. The concept is the assessment of the contractions of 2 muscle groups around a joint moving at a constant angular velocity in several repetitions. Given that there is an inverse relationship between strength of isokinetic contraction and angular velocity, lower velocities are used to measure the maximum strength and higher velocities (with a higher number of repetitions) to indicate the muscular endurance. Furthermore, the software delivers other measurements such as the overall work, movement amplitude, fatigue index, and the agonistic/antagonistic muscle groups' ratio ${ }^{10}$. These parameters allow defining the muscular strength profile (MSP) which is a reliable data in order to allow monitoring the individual strength training program for each athlete, and the rehabilitation of muscular injuries, mainly occurring in the lower limb ${ }^{11}$. Actually, it has been proven that the use of isokinetic equipment in top-level sports contributes to improving performance ${ }^{12}$. Technical performance parameters increase when the training succeeds in boosting the muscles strength and balance ${ }^{13}$. Rather, lack of strength and/or muscle imbalance affect negatively the performance and may lead to muscle injuries. Although not specific to judo 
movements, isokinetic muscular assessments seem to be important to establish relations of torque or strength among muscles with antagonist actions as well as different speeds of movement. In this context, recent research finds out a number of specifications related to judo. Due to the fact that one of the legs operates as a "supporting leg" while the other is always ready to attack the opponent, "attacking leg", there should be an imbalance between the muscle groups strengths in each $\mathrm{leg}^{4}$. Sport scientists are constantly researching new protocols adapted to each sport's specific motions and abilities ${ }^{5,14-16}$. To the best of the authors' knowledge, data about isokinetic strength of judo athletes are very few and showed conflicting results 4,5 . Moreover, it seems to be no research study in relation to elite judo African athletes. Thus, the purpose of this study conducted among 10 elites Tunisian judo athletes was to evaluate their MSP through 2 parameters: the muscular peak torque and the balance agonist/antagonist of the extensors and flexors of the knee and the external and internal rotators of the shoulder.

\section{Methods}

\section{Sample}

Ten athletes of the Tunisian male national judo team, one of the best teams in Africa, 7 times African champions, 2 times Mediterranean champions, silver medalists of the last African championship in 2012 and regular participants to the world cup and the Olympic games. Table 1 shows the main physiological characteristics of the study' participants.

\section{Instrumentation}

Participants underwent a muscular isokinetic assessment. The left and right knee muscles (Hamstring and Quadriceps) as well as the muscles rotating the shoulder (external and internal rotators) were tested using an isokinetic machine Cybex Humac Norm, testing and rehabilitation system in the Tunisian national center of sciences and medicine in sports.

The assessments of those 2 joints were performed randomly in separated days with only one joint test session per day.

\section{Testing}

Prior to the assessment, players performed a warmup in the gym consisting of 10 minutes on ergometric cycle, many sets of various dynamic repetitions of thigh and shoulder muscles, followed by standard stretching exercises.

We started with the knee testing. Each athlete was positioned in the machine with a hip fixed at $90^{\circ}$, and the dynamometer and knee joint axes were aligned. Strapping was used over the shank, thigh and waist to mini-
Table 1. Characteristics of the selected athletes of Tunisian male national judo team.

\begin{tabular}{ll}
\hline Age & $25.1 \pm 3,7$ years \\
Height & $178 \pm 9.4 \mathrm{~cm}$ \\
Weight & $79.4 \pm 13.7 \mathrm{Kg}$ \\
BMl & $24.6 \pm 1.8 \mathrm{Kg} / \mathrm{m}^{2}$ \\
Body fat & $11.0 \pm 2.4 \%$ \\
Muscle mass & $37.2 \pm 3.1 \mathrm{Kg}$ \\
Arm dominance & 7 Right \& 3 Left \\
\hline
\end{tabular}

mize secondary joint movements. A standard range of motion limit of $0^{\circ}$ to $120^{\circ}$ was passively set on the machine. Before starting the evaluation, athletes performed a familiarization session at the same velocities used in the test to minimize the learning effect and to ensure the reproducibility of the collected values. A Standardized testing protocol was followed at 3 different angular velocities, namely 90,180 , and $240 \%$ s.

Concerning shoulders, evaluation was performed in a seated position; each athlete was positioned on the machine uniformly as described in the Cybex® owner's manual for shoulder internal/external rotation. It was used many parts to accomplish the testing: a wrist/shoulder adapter, a lumbar cushion and many pad stabilizers for elbow, chest and pelvis. The dynamometer's axis of rotation was aligned with the longitudinal axis of the arm, the elbow was supported in $90^{\circ}$ flexion, the forearm and wrist were in neutral pronation/supination, the testing range of motion was $140^{\circ}$ composed of $80^{\circ}$ for internal rotation and $60^{\circ}$ for external rotation, from a reference position of the forearm horizontal at $0^{\circ}$.

During this muscular isokinetic assessment, all muscles performed concentric contractions and the studied parameters were calculated by the software of the dynamometer itself.

\section{Statistical analysis}

Data were presented as mean \pm Standard Deviation values. A paired samples Student's t-test was used to compare the difference of 2 parameters: (peak torque and ratio) between the arm dominant and non-dominant sides.

\section{Results}

In the present study, in all testing velocities, for knee and shoulder muscles groups, we focused on 2 values of measurements among many others delivered by the dynamometer's computer: maximum peak torque (PT) of each muscle (registered at the lowest velocity) to assess the strength and the concentric ratio agonistic/antagonistic muscles $(R)$ to evaluate the muscular balance.

The results of the knee assessment are presented in Table 2. They show a higher maximum PT of the quadriceps (knee extensors) in the opposite dominant arm 
side; the extensors of the "supporting leg" are significantly stronger than those of the "attacking leg" (245 N.m versus 237, $p<0.05$ ). The PT of Hamstrings (Knee flexors) was slightly higher in the supporting-leg (147 N.m versus $145, p>0.05$ ). The ratio Hamstring/quadriceps was normal at the 3 velocities for both legs (an average of $63 \%$ in both legs at the 3 speeds).

The shoulder evaluation, presented in Table 3 , showed a significant higher maximum $\mathrm{PT}$ of both shoulder rotators in the dominant arm side (84 N.m versus 71 for the internal rotators and 34,7 N.m versus 29,0 for the lateral rotators, $\mathrm{p}<0.05)$. Inversely, the ratio concentric external/internal rotators was higher in the non-dominant side $45 \%$ versus $35, \mathrm{p}<0 / 05$.

\section{Discussion}

The principal aim of the present study was to determine the muscular strength profile (MSP) of the Tunisian international judo athletes. To achieve this goal we used an isokinetic dynamometer. This machine provides the measurement of 3 types of muscular contractions-isometric, concentric isokinetic and eccentric isokinetic. It allows the assessment of various joint groups' muscles of lower limb, upper limb and trunk. In the field of sports medicine, the most commonly assessed joint is the knee, following a standardized protocol of 3 angular velocities low, intermediate and high, respectively 60 (or $90), 180$ and $240 \% / \mathrm{s}^{5,11,14,17}$. In this study, the performances of hamstrings and quadriceps were measured by the dynamometer during concentric contraction of both at 90,180 and $240 \%$ s.

Isokinetic assessment of shoulder remains a difficult issue because it's a joint with extensive mobility ${ }^{18,19}$. There is no consensus protocol20, a number of positions have been described in the literature with the seated position and $45^{\circ}$ of shoulder abduction in the scapular plane $20-22$. This position is reported to be more physiological, with a good reliability when assessing internal and external rotators ${ }^{20}$. The muscles groups were assessed during concentric contraction, at 2 angular speeds $(60 \%$ s and $120 \%$ s).

The isokinetic dynamometer delivers many measurements assessing the muscular strength profile (MSP) ${ }^{10,11,17}$. In the present study we used two of these parameters; the maximum peak torque of each muscle, in N.m and the ratio agonisitic/antagonistic muscles in \%.

Table 2. Peak torque (PT) of knee flexors and extensors and their ratio (R) at three angular velocities for dominant and non-dominant arm sides in selected athletes of Tunisian male national judo team.cet.

\begin{tabular}{|c|c|c|c|}
\hline & Dominant arm side & Non-dominant arm side & $P$ \\
\hline \multicolumn{4}{|c|}{ Flexors PT (Nm) } \\
\hline PT at. $90 \% / s$ & $145( \pm 17.4)$ & $147( \pm 18.7)$ & 0.729 \\
\hline PT at. $180 \% / s$ & $116( \pm 14.0)$ & $120( \pm 14.7)$ & 0.097 \\
\hline PT at. $240 \% / s$ & $103( \pm 16.6)$ & $107( \pm 14.4)$ & 0.208 \\
\hline \multicolumn{4}{|c|}{ Extensors PT (N.m) } \\
\hline PT at. $90 \%$ s & $237( \pm 31.6)$ & $245( \pm 28.7)$ & 0.013 \\
\hline PT at. $180 \% / \mathrm{s}$ & $182( \pm 23.4)$ & $188( \pm 21.7)$ & 0.018 \\
\hline PT at. $240 \% / s$ & $16.0( \pm 21.6)$ & $166( \pm 22.9)$ & 0.160 \\
\hline \multicolumn{4}{|l|}{ F/E Ratio (\%) } \\
\hline $\mathrm{R}$ at. $90 \% \mathrm{~s}$ & $61.9( \pm 6.87)$ & $60.7( \pm 7.79)$ & 0.332 \\
\hline $\mathrm{R}$ at. $180 \% / \mathrm{s}$ & $64.0( \pm 5.21)$ & $63.9( \pm 6.29)$ & 0.939 \\
\hline R at. $240 \% / s$ & $64.7( \pm 6.95)$ & $65.2( \pm 6.23)$ & 0.577 \\
\hline
\end{tabular}

Table 3. Peak torque (PT) of shoulder lateral and medial rotators and their ratio (R) at tow angular speeds for dominant and non-dominant arm sides in selected athletes of Tunisian male national judo team.cet.

\begin{tabular}{llll}
\hline & Dominant arm side & Non-dominant arm side & $p$ \\
\hline Lateral Rotators PT (N.m) & & & \\
\hline PT at. $60 \%$ s & $34,7( \pm 4.06)$ & $29.0( \pm 5.56)$ & 0.003 \\
PT at. $120 \%$ & $29.7( \pm 3.50)$ & $25.2( \pm 4.05)$ & 0.001 \\
\hline Medial Rotators PT (N.m) & & $71.1( \pm 8.58)$ & 0.000 \\
\hline PT at. $60 \%$ & $84.0( \pm 11.0)$ & $64.8( \pm 7.24)$ & 0.001 \\
PT at. $120 \%$ & $76.4( \pm 10.4)$ & & 0.000 \\
\hline L/M Rotators Ratio R $(\%)$ & & $46.8( \pm 4.97)$ & 0.001 \\
\hline R at. $90 \%$ s.s & $36.3( \pm 5.23)$ & $44.1( \pm 4.32)$ & \\
R at. $180 \%$ & $35.4( \pm 4.13)$ & & \\
\hline
\end{tabular}


The thigh muscles profile was characterized by a hamstrings' strength slightly higher and a Quadriceps' strength significantly higher; in the "supporting leg" than in the "attacking-leg". In Tunisian Judo internationals, the maximum Quadriceps PT was 245 N.m. at an angular velocity of $90 \%$ s., Andrade et al. ${ }^{5}$, registered $281 \mathrm{~N} . \mathrm{m}$ at $60 \%$ in Brazilian Olympic judo team and Drid et al. ${ }^{4}$ registered 270 N.m at $90 \%$ in Serbian national judo team. Among professional European and Brazilian footballers, this parameter was 270 N.m at $90^{\circ} / \mathrm{s}^{13,17}$.

The second parameter; ratio hamstrings/quadriceps $(H / Q)$, was $62 \%$, for both thighs. According to the literature, for an $\mathrm{H} / \mathrm{Q}$ ratio comprised between 55 and $65 \%$, there is no muscular imbalance requiring a specific strength program 4,5,11-13,15-17. European and Brazilian footballers have shown an average ratio of $64 \%{ }^{13,17}$. However, in Judo this parameter was not only little examined but the few results at hand were contradictory. Andrade found a ratio of $57 \%$ in Brazilian Olympic male team ${ }^{5}$, Drid on the other hand found an abnormally low ratio of $40,54 \% 4$. His results show a lack of hamstring strength among Serbian male national team; $110 \mathrm{~N} . \mathrm{m}$ at $60 \%$ s versus $163 \mathrm{N.m}$ at $90 \%$ for Andrade and $145 \mathrm{~N} . \mathrm{m}$ at $90 \% \mathrm{~s}$ in our study. Drid claims that this imbalance is due to the specificity of judo technique, since a judo contestant bears his weight on his standing leg, while he uses the other leg to throw the opponent to the ground. In top-level sport, we noticed that many authors favor the functional ratio $\mathrm{H}$. excentric/Q. concentric instead of the conventional one, $\mathrm{H}$. concentric/Q. concentric ${ }^{17}$.

The assessment of the shoulder rotators pointed out significantly stronger rotators of the dominant arm, with a maximum PT of $84 \mathrm{~N} . \mathrm{m}$ at $60 \%$ s for the internal rotators. Ribeiro et al. ${ }^{7}$ had used a Biodex machine to register $71 \mathrm{~N} . \mathrm{m}$ at $90 \%$ in Brazilian elite judo players. Similarly, Silva et al. ${ }^{8}$ noted $87 \mathrm{N.m}$ at $90 \%$ in Brazilian Paralympics team. Accordingly, Ichinose et al. ${ }^{9}$ assessed elbow extensors at $60 \%$ in Japanese national judo team using a cybexll, he found a significant difference in maximum PT (215 N.m versus 188 N.m) in the dominant arm. The ratio concentric external/internal rotators was higher in the non-dominant side $45 \%$ versus $35, p<0.05$ ), because of the high strength of internal rotators in judo ${ }^{7,8}$.

\section{Conclusion}

Muscle strength is a determinant factor in judo. However, little data exist relating to African athletes. In the present study we assessed the muscular strength profile (MSP) based on two isokinetic parameters obtained after the assessment of the muscles' groups of the knees and the shoulders.

The MSP of the selected elites Tunisian judo athletes was characterized by 3 major features; a strength of the shoulders' rotators higher in the dominant side, a strength of the quadriceps muscles in the standing leg significantly higher than in the attacking leg and a normal muscular balance Hamstrings/quadriceps in both legs. Regarding this last parameter, the literature related to Judo is limited and contradictory. More research is necessary to clarify this issue.

Data obtained in this study were used in twofold objectives; technical, by educating athletes and technicians on the need to rigorously strength train to maintain the ideal muscle profile and medical, by establishing a reference for the prevention and rehabilitation of lower limb muscles injuries. This study contributed to highlight data from a limited top-level sport population little studied so far $^{22}$. More studies are needed with a larger sample to allow better comparison with international data.

\section{References}

1. Callister R, Callister RJ, Staron RS, Fleck SJ, Tesch P, Dudley GA. Physiological characteristics of elite judo athletes. Int $J$ Sports Med. 1991;12:196-203.

2. Thomas SG, Cox MH, LeGal YM, Verde TJ, Smith HK. Physiological profiles of the Canadian National Judo Team. Can J Sport Sci. 1989;14:142-147.

3. Franchini E, Del Vecchio FB, Matsushigue KA, Guilherme G, Artioli G. Physiological Profiles of Elite Judo Athletes. Sports Medicine. 2011; 41, Issue 2;147-166.

4. Drid P, Drapsin M, Trivic T, Bratic M, Obadov S. Thigh muscles flexion/extension ratio in elite judo players. Journal of Combat Sports and Martial Arts. 2010; 1; Vol.1;21-25.

5. Andrade M, De Lira C, Koffes F, Mascarin N, Benedito-Silva A, Da Silva A. Isokinetic hamstrings-to-quadriceps peak torque ratio: the influence of sport modality, gender, and angular velocity. J Sports Sci. 2012;30:547-553.

6. Detanico D, Budal Arins F, Dal Pupo J, Dos Santos SG. Strength parameters in judo athletes: an approach using hand dominance and weight categories. Human Movement. 2012;vol.13:330-336.

7. Ribeiro SR, Tierra-Criollo CJ, Lopes Martins RB. Effects of different strengths in the judo fights, muscular electrical activity and biomechanical parameters in elite athletes. Rev Bras Med Esporte. 2006; Vol. 12, № 1.

8. Silva AC, Andrade MS. Avaliação isocinética em atletas paraolímpicos. Rev Bras Med Esporte. 2002; Vol. 8, № 3.

9. Ichinose $\mathrm{Y}, \mathrm{Kanehisa} \mathrm{H}$, Ito M, Kawakami Y, Fukunaga T. Relationship between muscle fiber pennation and force generation capability in Olympic athletes. Int J Sports Med. 1998; 19:541-546.

10. Baltzapoulos V, Brodie DA. Isokinetic dynamometry. Applications and limitations. Sports medicine. 1982;8:101-116.

11. Ghrairi $M$, Chomier $P$, Khelifa M, Ferret JM. Isokinetic strength and ratio of professional football players in UAE. Br J Sports Med. 2013;47:e3.

12. Tsuchiya Y, Ochi E, Sakuraba K, Kikuchi N, Hwang I. Isokinetic strength and anaerobic/intermittent capacity of Japenese lacrosse players. Isokinetics and Exercice Science. 2013; Vol. 21, N.1:77-82.

13. Eniseler N, Sahan C, Vurgum H, Mavi HF. Isokinetic Strength Responses to Season-long Training and Competition in Turkish Elite Soccer Players. J Hum Kinet. 2012;31:159-168.

14. Erwin Koninckx E, Van Leemputte M, Hespel P. Effect of isokinetic cycling versus weight training on maximal power output and endurance performance in cycling. European Journal of Applied Physiology. 2010; Vol. 09, Issue 4;699-708.

15. Harrison B, Firth W, Rogers S, et al. The relationship between isokinetic performance of hip and knee and jump perfor- 
mance in university rugby players. Isokinetics and Exercise Science. 2013;Volume 21, N. 2:175-180.

16. Padulo J, Oliva F, Frizziero A, Maffulli N. Muscles, Ligaments and Tendons Journal. Basic principles and recommendations in clinical and field science research. MLTJ. 2013;4:250-252.

17. Croisier JL, Reveillon V, Ferret JM, et al. Isokinetic assessment of knee flexors and extensors in professional soccer players. Isokinetics and Exercise Science. 2003;11:61-62.

18. Forthomme B, Crielaard JM, Croisier JL. Concept d'équilibre agonistes/antagonistes de l'épaule: analyse critique. In: Exercice musculaire excentrique. Masson Ed. 2009 ;61-68.

19. Codine P, Bernard PL, Pocholle M, Herisson C. Evaluation et rééducation des muscles de l'épaule en isocinétisme: métho- dologie, résultats et applications. Annales de réadaptation et de médecine physique. 2005;48:80-92.

20. Edouard P, Philippe B, Codinec D, et al. Reliability of shoulder rotators isokinetic strength imbalance measured using the Biodex dynamometer. Journal of Science and Medicine in Sport. 2013;16:162-165.

21. Hadzic V, Kalc M, Dervisevic E. Reproducibility of shoulder short range of motion in isokinetic and isometric strength testing. Journal of Exercise Science \& Fitness. 2012;10, Issue 2;83-89.

22. Yen D. Limitations of Isokinetic Testing to Determine Shoulder Strength after Rotator Cuff Repair. lowa Orthop J. 2005;25: 141-144. 